\title{
Associations of military divorce with mental, behavioral, and physical health outcomes
}

\author{
Lawrence Wang $^{1 *}$, Amber Seelig ${ }^{1}$, Shelley MacDermid Wadsworth² ${ }^{2}$ Hope McMaster ${ }^{1}$, John E. Alcaraz ${ }^{3}$ \\ and Nancy F. Crum-Cianflone ${ }^{1}$
}

\begin{abstract}
Background: Divorce has been linked with poor physical and mental health outcomes among civilians. Given the unique stressors experienced by U.S. service members, including lengthy and/or multiple deployments, this study aimed to examine the associations of recent divorce on health and military outcomes among a cohort of U.S. service members.

Methods: Millennium Cohort participants from the first enrollment panel, married at baseline (2001-2003), and married or divorced at follow-up (2004-2006), $(N=29,314)$. Those divorced were compared to those who remained married for mental, behavioral, physical health, and military outcomes using logistic regression models.

Results: Compared to those who remained married, recently divorced participants were significantly more likely to screen positive for new-onset posttraumatic stress disorder, depression, smoking initiation, binge drinking, alcohol-related problems, and experience moderate weight gain. However, they were also more likely be in the highest $15^{\text {th }}$ percentile of physical functioning, and be able to deploy within the subsequent 3-year period after divorce.

Conclusions: Recent divorce among military members was associated with adverse mental health outcomes and risky behaviors, but was also associated with higher odds of subsequent deployment. Attention should be given to those recently divorced regarding mental health and substance abuse treatment and prevention strategies.
\end{abstract}

\section{Background}

The associations between divorce and mental and physical health disorders have been investigated in civilian populations [1-9], demonstrating that divorce is related to lower health-related quality of life [2], and greater disability and mortality $[3,4]$. Recent research has shown that divorce is a risk factor for depression, posttraumatic stress disorder (PTSD), and elevated resting blood pressure, and is associated with other disorders such as seasonal affective disorder, social phobias, and bipolar disorder [5-7, 9]. Conversely, divorce has been linked to increased physical fitness in men [8]. Together, these findings present a clear picture of the physical and

\footnotetext{
* Correspondence: Lawrence.wang@med.navy.mil

'Deployment Health Research Department, Naval Health Research Center,

San Diego, CA, USA

Full list of author information is available at the end of the article
}

mental detriments associated with divorce among a civilian population.

Since the initiation of military operations in Iraq and Afghanistan after September $11^{\text {th }}$, the increased pace of military deployments has been shown to provide a protective effect against divorce [10] while at the same time, increasing the stress of military life and impacting the quality of marital relationships [11-13]. These differing results indicate that the relationship between deployment to Iraq and Afghanistan and divorce is not clear and additional studies are warranted [10, 14, 15]. Recent studies have demonstrated that infidelity and consideration of separation/divorce among military couples have increased during the recent conflicts $[14,15]$. Further, service members returning from the war with PTSD or serious injuries, including traumatic brain injury, can strain the marital bond [12]. Overall, these data suggest 
the importance of exploring the potential effects of divorce on health and military outcomes among service members during an era of persistent conflicts, because these outcomes not only affect quality of life, but also military force readiness [16]. The purpose of this investigation was to utilize a large, representative military cohort, using a prospective design to study the extent to which participants experiencing a recent divorce reported subsequent poor health and military outcomes compared to those who remained married during the same time frame. We hypothesized that, among military, recent divorce is associated with worsened mental and behavioral health, improved physical capability and would have a negative association with deployment and military separation.

\section{Methods}

\section{Population and data sources}

The Millennium Cohort Study was designed to prospectively assess the short- and long-term health outcomes of service members, and was initiated in 2001 prior to September $11^{\text {th }}$. Individuals invited to participate in the Millennium Cohort Study were randomly selected from US military rosters with oversampling of selected subgroups including women, Reserve and National Guard, and those who previously deployed (i.e., 1991 Gulf War or Bosnia, Kosovo, or Southwest Asia between 1998 and 2000) $[17,18]$. Participants include active duty, Reserve, and National Guard personnel from all five service branches who voluntarily agreed to complete follow-up surveys approximately every 3 years. The first panel of participants were enrolled during 2001-2003 ( $n=77,047)$, of whom 55,021 (71\%) completed a follow-up survey during 2004-2006. Additional details on Millennium Cohort Study methodology and response rates are described elsewhere $[16,17,19,20]$.

The current study evaluated participants in the first panel who were married at baseline and who were either married or divorced at the time of the follow-up survey $(n=34,500)$. Since military records were used to confirm marital status, participants who separated from military service before completing both surveys were excluded $(n=3419)$. In addition, participants missing demographic covariates (sex, birth year, education, race/ethnicity, $n=26$ ), missing military covariates (combat deployment, prior deployment, service component, service branch, pay grade, military occupation $n=707$ ), and with incomplete baseline physical, mental, or behavioral data (history of potential alcohol dependence, smoking status, body mass index, mental and physical functioning, $n=1034$ ) were excluded. This study was approved by the San Diego State University and Naval Health Research Center Institutional Review
Boards and written informed consent was obtained from all study participants.

Demographic and military data were obtained from electronic military personnel files, provided monthly by the Defense Manpower Data Center (DMDC). These files provided data on marital status, sex, birth date, race/ethnicity, education, service branch, service component, military pay grade, military occupation, dates of deployment, and date of military separation. Selfreported data collected on the Millennium Cohort Study questionnaires was used to assess all other variables in the analyses, including combat experiences.

\section{Outcome measures}

Specific mental, physical, behavioral, and functional health outcomes were selected based on previous findings regarding their impact on force health and readiness $[2-6,21]$. New-onset outcomes of interest were assessed only among those with no symptoms at baseline. Mental health outcomes included new-onset PTSD, new-onset depression, and anxiety/panic. The PTSD ChecklistCivilian Version (PCL-C), a 17-item symptom screening tool, was used to identify participants who screened positive for PTSD [22-25]. The PCL-C was scored using the specific criteria, where those who scored 50 or more on the PCL screened positive for PTSD symptoms. Participants screening positive for new-onset depression and anxiety/panic were identified using the PRIME-MD Patient Health Questionnaire (PHQ) [26-29]. The PHQ is a standardized tool to screen for mental health disorders based on the Diagnostic and Statistical Manual of Mental Disorders, 4th Edition.

Physical health outcomes included new-onset hypertension, new-onset diabetes, and weight change. Participants with diabetes and hypertension were identified using self-reported data on the Millennium Cohort questionnaire, where participants indicated a diagnosis from a health care professional. Weight change, determined using self-reported weight at baseline and follow-up, was calculated as the percent change from baseline to follow-up, and was categorized into five levels: extreme gain ( $>10 \%)$, moderate gain (between 3 and $10 \%)$, stable weight (within $3 \%$ of baseline weight), moderate loss (between 3 and $10 \%$ ), and extreme loss (>10\%) [30].

Behavioral outcomes included new-onset alcoholrelated problems, new-onset binge drinking, smoking initiation, and smoking recidivism. Alcohol-related problems were identified using the PHQ as making poor decisions regarding alcohol, such as driving under the influence or being hung over at work [27-29]. Binge drinking was defined as self-report of drinking five or more drinks on one occasion for men, and four or more drinks on one occasion for women [31]. Smoking 
initiation was defined as reporting current smoking at follow-up was assessed among baseline nonsmokers, and smoking recidivism, defined as report of current smoking at follow-up was assessed among baseline former smokers.

Functional health outcomes, assessed using the Mental Component Summary (MCS) score and Physical Component Summary (PCS) score, were included to portray a general assessment of mental and physical health status at follow-up. The MCS and PCS were calculated using the Medical Outcomes Study Short Form 36-item Survey for Veterans (SF-36 V), a measure designed to evaluate physical and mental functioning $[32,33]$. Both MCS and PCS scores were categorized as one) lowest $15^{\text {th }}$ percentile, two) middle $70^{\text {th }}$ percentile, and three) highest $15^{\text {th }}$ percentile, where a higher score represents better functioning. The resulting groups correspond approximately to participants scoring more than one standard deviation below, within one standard deviation of, and more than one standard deviation above the mean.

Military-specific outcomes from the DMDC included deployment and separation from the military. Deployment (yes/no) was assessed during the 3-year period between the baseline and first follow-up survey in the Stayed Married group, and during the 3-year period following divorce for the Recently Divorced group. While the timing is different for each groups, the length of follow-up during which they had the opportunity to have the outcome (deployment) is the same. This method was chosen to ensure that the outcome occurred following the exposure, and that the time from exposure to outcome was the same. Separation from the military was assessed during the 3 years after the follow-up survey was completed for both groups, since all participants in these analyses remained in the military through their follow-up survey.

\section{Additional variables}

Baseline characteristics were included in the analyses to adjust for factors as suggested by previous studies $[19,29,34-49]$. Variables included sex, birth year, education, race/ethnicity, military pay grade, service component, service branch, and military occupation. These variables were determined using DMDC records and were backfilled with self-reported data to reduce missing values. Baseline functional health was evaluated with the MCS and PCS using the categorization as described above. A prior history of potential alcohol dependence was assessed at baseline using the CAGE questionnaire (Cutting down, Annoyance by criticism, Guilty feeling, and Eye-opener); those that were categorized as having a history of potential alcohol dependence positively endorsed at least one of the CAGE questions [50, 51].
Baseline smoking status was determined using self-report, where current smokers were defined as those who indicated smoking at least 100 cigarettes in their lifetime and had either not tried to quit or had been unsuccessful at quitting. Baseline body mass index (BMI) was categorized as underweight $\left(<18.5 \mathrm{~kg} / \mathrm{m}^{2}\right)$, normal range (18.5-24.9), overweight (25-29.9), or obese ( $\geq 30.0)$ [37].

Combat experience was defined as having personally witnessed at least one of the following events: death due to war, disaster or tragic event; physical abuse; dead or decomposing bodies; maimed soldiers or civilians; or prisoners of war or refugees. Combat was assessed at follow-up among deployers and was reported as having occurred during the same 3-year period as a deployment. Participants were categorized at baseline as deployed with combat experiences, deployed without combat experiences, and non-deployed. An additional deployment variable was included, describing deployment prior to 2001 and was assessed using military personnel records.

\section{Statistical analyses}

Chi-square tests of association and unadjusted logistic regression models were used to compare characteristics between divorced and married service members. In multivariable analyses, separate logistic regression models were utilized to compare the odds of each outcome in relation to marital status while adjusting for relevant covariates. Polychotomous logistic regression was used to assess the tiered outcomes including weight change, MCS, and PCS. Multicollinearity was evaluated among all independent variables using a variance inflation factor of four. Final models were determined using a change in estimate approach, where variables were retained if significant $(\mathrm{p}<0.05)$ or confounders (defined as a $10 \%$ change in adjusted odds ratio). Sex and combat deployment were a priori maintained in the adjusted models. Based on this, the final models included sex, birth year, education, race/ethnicity, baseline smoking status, history of potential alcohol dependence, body mass index, baseline MCS and PCS, combat deployment, pay grade, service component, service branch, and occupation. Subanalyses were performed among service members divorced during follow-up, in which those who were divorced for $>2$ years at their follow-up survey (reference group) were compared to those divorced for $<1$ year, and those divorced for 1-2 years. Data management and statistical analyses were performed using SAS, version 9.3, statistical software (SAS Institute, Inc., Cary, North Carolina).

\section{Results}

During the study period, 1545 (5.3 \%) service members became divorced and 27,769 remained married. Service 
Table 1 Baseline demographic, military, and behavioral characteristics by marital status ${ }^{a}$

\begin{tabular}{|c|c|c|c|c|c|c|}
\hline \multirow[b]{3}{*}{ Baseline characteristics $^{b}$} & \multirow{2}{*}{\multicolumn{2}{|c|}{$\begin{array}{l}\text { Recently divorced } \\
n=1545\end{array}$}} & \multirow{2}{*}{\multicolumn{2}{|c|}{$\begin{array}{l}\text { Stayed married } \\
n=27,769\end{array}$}} & \multirow{2}{*}{\multicolumn{2}{|c|}{$\begin{array}{l}\text { Total } \\
n=29,314\end{array}$}} \\
\hline & & & & & & \\
\hline & $\mathrm{n}$ & (\%) & $\bar{n}$ & (\%) & $\mathrm{n}$ & (\%) \\
\hline \multicolumn{7}{|l|}{ Sex } \\
\hline Male & 959 & 62.07 & 22800 & 82.11 & 23759 & 81.05 \\
\hline Female & 586 & 37.93 & 4969 & 17.89 & 5555 & 18.95 \\
\hline \multicolumn{7}{|l|}{ Age } \\
\hline Born before 1960 & 236 & 15.28 & 7581 & 27.30 & 7817 & 26.67 \\
\hline 1960-1969 & 668 & 43.24 & 13195 & 47.52 & 13863 & 47.29 \\
\hline 1970-1979 & 599 & 38.77 & 6767 & 24.37 & 7366 & 25.13 \\
\hline 1980 and after & 42 & 2.72 & 226 & 0.81 & 268 & 0.91 \\
\hline \multicolumn{7}{|l|}{ Education } \\
\hline Some college or less & 1212 & 78.45 & 18289 & 65.86 & 19501 & 66.52 \\
\hline Bachelor's degree or higher & 333 & 21.55 & 9480 & 34.14 & 9813 & 33.48 \\
\hline \multicolumn{7}{|l|}{ Race/Ethnicity } \\
\hline White, non-Hispanic & 1098 & 71.07 & 20171 & 72.64 & 21269 & 72.56 \\
\hline Black, non-Hispanic & 214 & 13.85 & 2853 & 10.27 & 3067 & 10.46 \\
\hline Other & 233 & 15.08 & 4745 & 17.09 & 4978 & 16.98 \\
\hline \multicolumn{7}{|l|}{ Combat deployment } \\
\hline Not deployed & 1030 & 66.67 & 19076 & 68.70 & 20106 & 68.59 \\
\hline Deployed, without combat & 279 & 18.06 & 4345 & 15.65 & 4624 & 15.77 \\
\hline Deployed, with combat & 236 & 15.28 & 4348 & 15.66 & 4584 & 15.64 \\
\hline \multicolumn{7}{|l|}{ Prior deployment } \\
\hline Yes & 445 & 28.80 & 9053 & 32.60 & 9498 & 32.40 \\
\hline No & 1100 & 71.20 & 18716 & 67.40 & 19816 & 67.60 \\
\hline \multicolumn{7}{|l|}{ Military pay grade } \\
\hline Enlisted & 1263 & 81.75 & 18807 & 67.73 & 20070 & 68.47 \\
\hline Officer & 282 & 18.25 & 8962 & 32.27 & 9244 & 31.53 \\
\hline \multicolumn{7}{|l|}{ Service component } \\
\hline Active duty & 873 & 56.50 & 14951 & 53.84 & 15824 & 53.98 \\
\hline Reserve/National Guard & 672 & 43.50 & 12818 & 46.16 & 13490 & 46.02 \\
\hline \multicolumn{7}{|l|}{ Service branch } \\
\hline Army & 721 & 46.67 & 12940 & 46.60 & 13661 & 46.60 \\
\hline Navy/Coast Guard & 228 & 14.76 & 4373 & 15.75 & 4601 & 15.70 \\
\hline Air Force & 538 & 34.82 & 9432 & 33.97 & 9970 & 34.01 \\
\hline Marines & 58 & 3.75 & 1024 & 3.69 & 1082 & 3.69 \\
\hline \multicolumn{7}{|l|}{ Military occupation } \\
\hline Combat specialist & 273 & 17.67 & 6402 & 23.05 & 6675 & 22.77 \\
\hline Health care & 165 & 10.68 & 2997 & 10.79 & 3162 & 10.79 \\
\hline Other & 1107 & 71.65 & 18370 & 66.15 & 19477 & 66.44 \\
\hline \multicolumn{7}{|c|}{ History of potential alcohol dependence ${ }^{c}$} \\
\hline Yes & 296 & 19.16 & 4617 & 16.63 & 4913 & 16.76 \\
\hline No & 1249 & 80.84 & 23152 & 83.37 & 24401 & 83.24 \\
\hline
\end{tabular}


Table 1 Baseline demographic, military, and behavioral characteristics by marital status ${ }^{a}$ (Continued)

\begin{tabular}{|c|c|c|c|c|c|c|}
\hline \multicolumn{7}{|l|}{ Smoking status } \\
\hline Current smoker & 317 & 20.52 & 3958 & 14.25 & 4275 & 14.58 \\
\hline Other & 1228 & 79.48 & 23811 & 85.75 & 25039 & 85.42 \\
\hline \multicolumn{7}{|l|}{ Body mass index ${ }^{d}$} \\
\hline Underweight and normal weight & 701 & 45.37 & 8670 & 31.22 & 9371 & 31.97 \\
\hline Overweight & 717 & 46.41 & 15975 & 57.53 & 16692 & 56.94 \\
\hline Obese & 127 & 8.22 & 3124 & 11.25 & 3251 & 11.09 \\
\hline \multicolumn{7}{|l|}{ Mental component summary score $e^{\mathrm{e}}$} \\
\hline Lowest $15^{\text {th }}$ percentile & 296 & 19.16 & 2568 & 9.25 & 2864 & 9.77 \\
\hline Middle $70^{\text {th }}$ percentile & 1043 & 67.51 & 19553 & 70.41 & 20596 & 70.26 \\
\hline Highest $15^{\text {th }}$ percentile & 206 & 13.33 & 5648 & 20.34 & 5854 & 19.97 \\
\hline \multicolumn{7}{|l|}{ Physical component summary score $\mathrm{e}^{\mathrm{e}}$} \\
\hline Lowest $15^{\text {th }}$ percentile & 188 & 12.17 & 3604 & 12.98 & 3792 & 12.94 \\
\hline Middle $70^{\text {th }}$ percentile & 1065 & 68.93 & 20675 & 74.45 & 21740 & 74.16 \\
\hline Highest $15^{\text {th }}$ percentile & 292 & 18.90 & 3490 & 12.57 & 3782 & 12.90 \\
\hline
\end{tabular}

members who divorced were proportionally more likely at baseline to be female, younger, less educated, enlisted, active duty, under- or normal weight, and have lower mental and higher physical functioning compared to those who remained married (Table 1). All characteristics were significantly associated with the exposure at $p<0.05$, except for service branch $(p=0.74)$.

Table 2 displays the prevalence of each outcome by marital status. New-onset PTSD, depression, and anxiety were more prevalent among those recently divorced with $4.8 \%$, $3.0 \%$, and $3.0 \%$ developing each of these conditions, respectively, between baseline and follow-up. Smoking initiation and recidivism, alcohol-related problems, and binge drinking were also more prevalent in divorced service members compared to those who remained married. Regarding physical health, weight gain was more prevalent in the divorced group, and married individuals were more likely to have a stable weight. Divorced service members were also more likely to be in the lowest $15^{\text {th }}$ percentile for mental functioning, and be in the highest $15^{\text {th }}$ percentile for physical functioning. Finally, divorced service members were proportionately more likely to deploy within 3 years following divorce, but had similar percentages of military separation. All outcomes were significantly associated with exposure at $p<0.05$, except for new-onset diabetes $(p=0.08)$ and military separation $(p=0.33)$.
In the multivariable models (Table 3), recently divorced service members were more likely to screen positive for new-onset PTSD [Adjusted Odds Ratio (AOR) 1.77; $95 \%$ Confidence Interval (CI): 1.36, 2.30] and newonset depression (AOR: 1.40; $95 \% \mathrm{CI}: 1.02,1.94$ ) than those individuals who stayed married. Models focusing on behavioral health outcomes showed that those who were recently divorced were more likely to initiate smoking (AOR: 2.04; 95 \% CI: 1.18, 3.54), develop alcoholrelated problems (AOR: 1.52; $95 \% \mathrm{CI}: 1.15,2.00$ ), and initiate binge drinking (AOR: 1.51; $95 \% \mathrm{CI}: 1.26,1.81$ ) than those who remained married. Regarding physical health outcomes, those who were recently divorced were more likely to experience moderate (between $3 \%$ and $10 \%$ ) weight gain (AOR: 1.15; $95 \%$ CI: 1.02, 1.31) than those who remained married. Finally, service members who were recently divorced were more likely to be in the highest $15^{\text {th }}$ percentile of physical health functioning based on the PCS score (AOR: 1.26; 95 \% CI: 1.10, 1.46). After adjusting for other covariates in the reduced model, analyses examining deployment and military separation showed that those divorced were more likely to deploy (AOR:1.47; 95 \% CI: 1.31, 1.66) than those people who stayed married. Experiencing a recent divorce was not found to be significantly associated with separation from the military. 
Table 2 Prevalence of health and military outcomes by marital status ${ }^{\mathrm{a}}$

\begin{tabular}{|c|c|c|c|c|c|c|}
\hline \multirow[b]{3}{*}{ Outcome $^{b}$} & \multirow{2}{*}{\multicolumn{2}{|c|}{$\begin{array}{l}\text { Recently divorced } \\
N=1545\end{array}$}} & \multirow{2}{*}{\multicolumn{2}{|c|}{$\begin{array}{l}\text { Stayed married } \\
N=27,769\end{array}$}} & \multirow{2}{*}{\multicolumn{2}{|c|}{$\begin{array}{l}\text { Total } \\
N=29,314\end{array}$}} \\
\hline & & & & & & \\
\hline & $\mathrm{n}$ & $(\%)$ & $\mathrm{n}$ & $(\%)$ & $\mathrm{n}$ & $(\%)$ \\
\hline \multicolumn{7}{|l|}{ Mental health } \\
\hline New-onset posttraumatic stress disorder ${ }^{\mathrm{b}}$ & 74 & 4.79 & 597 & 2.15 & 671 & 2.29 \\
\hline New-onset depression ${ }^{c}$ & 47 & 3.04 & 431 & 1.55 & 478 & 1.63 \\
\hline Anxiety/Panic ${ }^{c}$ & 47 & 3.04 & 476 & 1.72 & 523 & 1.78 \\
\hline \multicolumn{7}{|l|}{ Behavioral } \\
\hline Smoking initiation & 15 & 0.97 & 125 & 0.45 & 140 & 0.48 \\
\hline Smoking recidivism & 53 & 3.43 & 685 & 2.47 & 738 & 2.52 \\
\hline New-onset alcohol-related problems ${ }^{c}$ & 62 & 4.01 & 727 & 2.62 & 789 & 2.69 \\
\hline New-onset binge drinking ${ }^{d}$ & 183 & 11.84 & 2846 & 10.25 & 3029 & 10.33 \\
\hline \multicolumn{7}{|l|}{ Physical health } \\
\hline New-onset hypertension & 84 & 5.44 & 1646 & 5.93 & 1730 & 5.90 \\
\hline New-onset diabetes & 8 & 0.52 & 205 & 0.74 & 213 & 0.73 \\
\hline \multicolumn{7}{|l|}{ Weight change ${ }^{e}$} \\
\hline Extreme weight loss & 46 & 2.98 & 572 & 2.06 & 618 & 2.11 \\
\hline Moderate weight loss & 169 & 10.94 & 3013 & 10.85 & 3182 & 10.85 \\
\hline Stable weight & 572 & 37.02 & 12490 & 44.98 & 13062 & 44.56 \\
\hline Moderate weight gain & 522 & 33.79 & 7949 & 28.63 & 8471 & 28.90 \\
\hline Extreme weight gain & 120 & 7.77 & 1634 & 5.88 & 1754 & 5.98 \\
\hline \multicolumn{7}{|l|}{ Functional health } \\
\hline \multicolumn{7}{|l|}{ Mental component summary score ${ }^{f}$} \\
\hline Lowest $15^{\text {th }}$ percentile & 242 & 15.66 & 2761 & 9.94 & 3003 & 10.24 \\
\hline Middle $70^{\text {th }}$ percentile & 1061 & 68.67 & 19563 & 70.45 & 20624 & 70.36 \\
\hline Highest $15^{\text {th }}$ percentile & 221 & 14.30 & 4999 & 18.00 & 5220 & 17.81 \\
\hline \multicolumn{7}{|l|}{ Physical component summary score ${ }^{f}$} \\
\hline Lowest $15^{\text {th }}$ percentile & 211 & 13.66 & 3633 & 13.08 & 3884 & 13.11 \\
\hline Middle $70^{\text {th }}$ percentile & 1031 & 66.73 & 19928 & 71.76 & 20959 & 71.50 \\
\hline Highest $15^{\text {th }}$ percentile & 282 & 18.25 & 3762 & 13.55 & 4044 & 13.80 \\
\hline \multicolumn{7}{|l|}{ Military related outcomes } \\
\hline Deployment ${ }^{9}$ & 541 & 38.26 & 8693 & 31.30 & 9234 & 31.64 \\
\hline Military separation ${ }^{\text {h }}$ & 205 & 13.27 & 3929 & 14.15 & 4134 & 14.10 \\
\hline
\end{tabular}

a Marital status assessed from baseline to first follow-up, where those who were "recently divorced" were married at their baseline survey and got divorced before their follow-up survey, those who "stayed married" were married throughout the study period. The reference category is "stayed married"

${ }^{\mathrm{b}}$ All outcomes were significantly associated with exposure at $p<0.05$, except for new-onset diabetes $(p=0.08)$ and military separation ( $p=0.33$ )

c Posttraumatic stress disorder is defined using the PTSD checklist-civilian version, specific criteria with a cutoff of 50

d Assessed using the Patient Health Questionnaire

e Binge drinking is defined as reporting 5 or more drinks per occasion for men or 4 or more drinks per occasion for women on at least 1 day during the past year

${ }^{f}$ Extreme weight loss ( $\geq 10 \%$ loss), moderate weight loss (between $3 \%$ and $10 \%$ loss), stable weight (within $\pm 3 \%$ ), moderate weight gain (between $3 \%$ and

$10 \%$ gain), and extreme weight gain ( $\geq 10 \%$ gain)

${ }^{9}$ Mental component summary score and physical component summary score are obtained from the short form 36 question health survey for veterans (SF-36 V)

h Deployment was assessed from baseline until the follow-up for those in the "stayed married" and in the 3 years following divorce for the "recently

divorced" group

' Separation was assessed after the date of the completion of the follow-up survey

Among divorced service members, we examined the association of the timing between the divorce and the assessment of health and military outcomes. We compared those who completed their survey within 1 year after their divorce date with those who had between 2 and 3 years between their divorce and completion of survey. The latter served as the reference group. In the adjusted model, those who completed the survey within 
Table 3 Adjusted $^{a}$ odds of health and military outcomes comparing recently divorced military personnel with those who stayed married ${ }^{b}$

\begin{tabular}{|c|c|c|}
\hline \multirow[b]{2}{*}{ Outcome $^{c}$} & \multicolumn{2}{|c|}{ Divorced vs. married } \\
\hline & $\overline{\mathrm{AOR}}$ & $(95 \% \mathrm{Cl})$ \\
\hline \multicolumn{3}{|l|}{ Mental health } \\
\hline New-onset posttraumatic stress disorder ${ }^{d}$ & $1.77^{k}$ & $(1.36,2.30)$ \\
\hline New-onset depression ${ }^{\mathrm{e}}$ & $1.40^{\mathrm{k}}$ & $(1.02,1.94)$ \\
\hline Anxiety/panic ${ }^{e}$ & 1.24 & $(0.90,1.71)$ \\
\hline \multicolumn{3}{|l|}{ Behavioral } \\
\hline Smoking initiation & $2.04^{k}$ & $(1.18,3.54)$ \\
\hline Smoking recidivism & 1.25 & $(0.93,1.68)$ \\
\hline New-onset alcohol-related problems ${ }^{\mathrm{e}}$ & $1.52^{\mathrm{k}}$ & $(1.15,2.00)$ \\
\hline New-onset binge drinking ${ }^{f}$ & $1.51^{\mathrm{k}}$ & $(1.26,1.81)$ \\
\hline \multicolumn{3}{|l|}{ Physical health } \\
\hline New-onset hypertension & 1.13 & $(0.89,1.42)$ \\
\hline New-onset diabetes & 0.82 & $(0.40,1.69)$ \\
\hline \multicolumn{3}{|l|}{ Weight change ${ }^{g}$} \\
\hline Extreme weight loss & 1.31 & $(0.94,1.80)$ \\
\hline Moderate weight loss & 1.08 & $(0.90,1.29)$ \\
\hline Stable weight & 1.00 & - \\
\hline Moderate weight gain & $1.15^{\mathrm{k}}$ & $(1.02,1.31)$ \\
\hline Extreme weight gain & 0.90 & $(0.73,1.12)$ \\
\hline \multicolumn{3}{|l|}{ Functional health } \\
\hline \multicolumn{3}{|l|}{ Mental component summary score ${ }^{\mathrm{h}}$} \\
\hline Lowest $1^{\text {th }}$ percentile & 1.09 & $(0.93,1.28)$ \\
\hline Middle $70^{\text {th }}$ percentile & 1.00 & - \\
\hline Highest $15^{\text {th }}$ percentile & 1.02 & $(0.87,1.19)$ \\
\hline \multicolumn{3}{|l|}{ Physical component summary score ${ }^{\text {h }}$} \\
\hline Lowest $15^{\text {th }}$ percentile & 1.01 & $(0.86,1.20)$ \\
\hline Middle $70^{\text {th }}$ percentile & 1.00 & - \\
\hline Highest $15^{\text {th }}$ percentile & $1.26^{\mathrm{k}}$ & $(1.10,1.46)$ \\
\hline \multicolumn{3}{|l|}{ Military related outcomes } \\
\hline Deployment' & $1.47^{k}$ & $(1.31,1.66)$ \\
\hline Military separation ${ }^{j}$ & 0.95 & $(0.80,1.11)$ \\
\hline
\end{tabular}

${ }^{a}$ Full models were adjusted for all variables: sex, birth year, education, race/ethnicity, baseline smoking status, history of potential alcohol dependence, body mass index, baseline MCS and PCS, combat deployment, pay grade, service component, service branch, and occupation. Reduction of models utilized backwards stepwise regression

${ }^{b}$ Marital status assessed from baseline to first follow-up, where those who were "recently divorced" were married at their baseline survey and got divorced before their follow-up survey, those who "stayed married" were married throughout the study period. The reference category is "stayed married"

"In outcomes without a reference indication, the reference category is "No"

d Posttraumatic stress disorder is defined using the PTSD checklist-civilian version, specific criteria with a cutoff of 50. ${ }^{e}$ Assessed using the Patient Health Questionnaire

${ }_{\mathrm{f}}$ Binge drinking is defined as reporting 5 or more drinks per occasion for men or 4 or more drinks per occasion for women on at least 1 day during the past year

${ }^{9}$ Extreme weight loss ( $\geq 10 \%$ loss), moderate weight loss (between $3 \%$ and $10 \%$ loss), stable weight (within $\pm 3 \%$ ), moderate weight gain (between $3 \%$ and $10 \%$ gain), and extreme weight gain ( $\geq 10 \%$ gain)

${ }^{\mathrm{h}}$ Mental component summary score and physical component summary score are obtained from the Short Form-36 Question Health Survey for Veterans (SF-36 V)

' Deployment was assessed from baseline until the follow-up for those in the "stayed married" and assessed until 3 years after divorce event for the "recently

divorced" population

j Separation was assessed after the date of the completion of the follow-up survey

${ }^{\mathrm{k}}$ Adjusted Odds Ratio was found to be statistically significant 
a year of their divorce date were significantly more likely to screen positive for new-onset PTSD (AOR: 3.27; $95 \%$ CI: 1.56, 6.86), have alcohol-related problems (AOR: 2.24; $95 \% \mathrm{CI}: 1.11,4.53$ ), and binge drinking (AOR: 1.90; 95 \% CI: 1.18, 3.06) compared to those who completed the survey between 2 and 3 years after the divorce date (Table 4). They were also less likely to report extreme weight gain (AOR: 0.41; 95 \% CI: 0.23, 0.71). We also compared those who had completed their survey between 1 and 2 years after their divorce. The reference group, those with between 2 and 3 years between their divorce and completion of survey, remained the same. In the adjusted model, those who had between 1 and 2 years since divorce were less likely to report binge drinking (AOR: 0.53;
95 \% CI: 0.31, 0.90) compared to those who completed the survey between 2 and 3 years after their divorce.

\section{Discussion}

This prospective study evaluated the associations of divorce on a comprehensive set of mental, behavioral, physical, and functional health outcomes in a large military cohort during the Iraq and Afghanistan wars. Data on the impact of divorce in a military setting are especially important given the increasing rates of marital distress associated with the recent conflicts $[10,12,14]$. After controlling for baseline sociodemographics and health status, this study showed significant associations between divorce and new-onset PTSD, depression, smoking initiation and

Table 4 Adjusted $^{a}$ odds of health and military outcomes by timing of divorce ${ }^{b}$

\begin{tabular}{|c|c|c|c|c|}
\hline \multirow[b]{2}{*}{ Outcome $^{c}$} & \multicolumn{2}{|c|}{$<1$ year since divorce } & \multicolumn{2}{|c|}{$1-2$ years since divorce } \\
\hline & $\overline{A O R}$ & $(95 \% \mathrm{Cl})$ & $\mathrm{AOR}$ & $(95 \% \mathrm{Cl})$ \\
\hline \multicolumn{5}{|l|}{ Mental health } \\
\hline New-onset posttraumatic stress disorder ${ }^{d}$ & $3.27^{\mathrm{j}}$ & $(1.56,6.86)$ & 1.79 & $(0.82,3.92)$ \\
\hline New-onset depression ${ }^{e}$ & 1.16 & $(0.53,2.53)$ & 1.19 & $(0.54,2.60)$ \\
\hline \multicolumn{5}{|l|}{ Behavioral } \\
\hline Smoking initiation & 0.83 & $(0.25,2.78)$ & 0.53 & $(0.14,2.04)$ \\
\hline New-onset alcohol-related problems ${ }^{\mathrm{e}}$ & $2.24^{j}$ & $(1.11,4.53)$ & 1.07 & $(0.50,2.29)$ \\
\hline New-onset binge drinking ${ }^{f}$ & $1.90^{\mathrm{j}}$ & $(1.18,3.06)$ & $0.53^{j}$ & $(0.31,0.90)$ \\
\hline \multicolumn{5}{|l|}{ Physical health } \\
\hline \multicolumn{5}{|l|}{ Weight change ${ }^{g}$} \\
\hline Extreme weight loss & 1.82 & $(0.80,4.14)$ & 0.93 & $(0.37,2.31)$ \\
\hline Moderate weight loss & 1.54 & $(0.97,2.43)$ & 0.93 & $(0.57,1.51)$ \\
\hline Stable weight & 1.00 & - & 1.00 & - \\
\hline Moderate weight gain & 0.85 & $(0.62,1.16)$ & 0.93 & $(0.69,1.27)$ \\
\hline Extreme weight gain & $0.41^{j}$ & $(0.23,0.71)$ & 0.87 & $(0.54,1.41)$ \\
\hline \multicolumn{5}{|l|}{ Functional health } \\
\hline \multicolumn{5}{|l|}{ Physical component summary score ${ }^{h}$} \\
\hline Lowest $15^{\text {th }}$ percentile & 0.86 & $(0.57,1.29)$ & 0.90 & $(0.60,1.34)$ \\
\hline Middle $70^{\text {th }}$ percentile & 1.00 & - & 1.00 & - \\
\hline Highest $15^{\text {th }}$ percentile & 1.32 & $(0.92,1.88)$ & 0.96 & $(0.67,1.38)$ \\
\hline \multicolumn{5}{|l|}{ Military related outcomes } \\
\hline Deployment ${ }^{i}$ & 1.17 & $(0.87,1.56)$ & 1.05 & $(0.79,1.40)$ \\
\hline \multicolumn{5}{|c|}{$\begin{array}{l}\text { a Full models were adjusted for all variables: sex, birth year, education, race/ethnicity, baseline smoking status, history of potential alcohol dependence, body mas } \\
\text { index, baseline MCS and PCS, combat deployment, pay grade, service component, service branch, occupation } \\
\text { b Marital status assessed from baseline to first follow-up, where those who were "recently divorced" were married at their baseline survey and got divorced befor } \\
\text { their follow-up survey. The reference category is }>2 \text { years since divorce } \\
\text { c In outcomes without a reference indication, the reference category is "No" } \\
\text { d Posttraumatic stress disorder is defined using the PTSD checklist-civilian version, specific criteria with a cutoff of } 50 \\
\text { e Assessed using the Patient Health Questionnaire } \\
\text { f Binge drinking is defined as reporting five or more drinks per occasion for men or four or more drinks per occasion for women on at least } 1 \text { day during the } \\
\text { past year } \\
g^{9} \text { Extreme weight loss ( } \geq 10 \% \text { loss), moderate weight loss (between } 3 \% \text { and } 10 \% \text { loss), stable weight (within } \pm 3 \% \text { ), moderate weight gain (between } 3 \% \text { and } \\
10 \% \text { gain), and extreme weight gain ( } \geq 10 \% \text { gain) } \\
\text { h Physical component summary score are obtained from the Short Form-36 Question Health Survey for Veterans (SF- } 36 \mathrm{~V} \text { ) } \\
\text { ' Deployment was assessed from baseline until the follow-up for those in the "stayed married" and assessed until } 3 \text { years after divorce event for the "recently } \\
\text { divorced" population } \\
\text { jAdjusted Odds Ratio was found to be statistically significant }\end{array}$} \\
\hline
\end{tabular}


recidivism, alcohol-related problems, binge drinking, and moderate weight gain. Study results suggest that special attention should be given to recently divorced individuals regarding mental health and risky health-related behaviors treatment and prevention strategies.

Studies of the consequences of divorce in the general population have revealed a variety of consequences for both adults and children. Although health implications for adults are in part due to selection effects, the bulk of the evidence attributes negative outcomes to the effects of divorce rather than selection [52]. Divorce can result in both acute and chronic stress for adult partners, with significant emotional upheaval that can include conflict, resentment, anger, and sadness [52]. In the military, divorce may mean loss of access to on-base housing or other changes in living arrangements, loss of medical benefits, and reduced family income as well as loss of spousal support and assistance. Although both shortand long-term consequences can occur, declines in wellbeing are temporary for most individuals [53].

This study found significant associations between divorce and the development of new-onset PTSD. Previous research regarding the relationship of divorce and PTSD has found bidirectional relationships. PTSD has been linked to elevated levels of hostile behavior, decreased capacity for intimacy, marital distress, and domestic violence $[54,55]$, all which may increase the likelihood of divorce. In a study examining male Vietnam veterans, those with PTSD had more problems in their relationships, more difficulties with intimacy, and had taken more steps toward separation and divorce than veterans not screening positive for PTSD [56]. Life events such as divorce may also generate symptoms of post-traumatic stress similar to other types of trauma such as accidents or abuse [57]. Our study results showed a relationship between divorce and screening positive for PTSD within a 3-year period when compared to those who remained married. This suggests that the mental health effects of divorce occur over a relatively short time period, information that may be helpful for providers caring for recently divorced individuals.

Similarly, our study found a significant association between divorce and depression, which is consistent with results from previous research, including a civilian study that found an association as early as 4-years [58], though our results show a shorter time frame of $\leq 3$ years. Of note, the association between divorce and depression was present when comparing recently divorced service members to those who remained married, but depression was similar among recent divorcees and those who were single. Understanding the temporal proximity of the association between a recent divorce and mental health issues may help enable faster detection and management of these conditions.
Alcohol-related problems are detrimental to overall occupational performance especially during military operations. The present study showed a statistically significant association between divorce and alcohol-related problems and binge drinking. Previous studies have shown that drinking problems increase the risk of divorce $[9,59]$. Our results, after controlling for sociodemographic factors and potential alcohol dependence at baseline, support an association between divorce and alcohol use. A longer follow-up period with evaluation of the temporal sequence could further elucidate the link between divorce and alcohol misuse. Our results also indicated that recent divorcees were significantly more likely to initiate smoking. These data support the hypothesis that marital support may be advantageous in reducing risky health-related behaviors [60].

The results of our study indicated a relationship between a recent divorce and improved physical function, and counterintuitively, also moderate weight gain. Given that there was no association with more extreme weight changes, and previous observational research has reported that divorce is associated with increased physical fitness in men [8], we hypothesize that divorce may be linked to increased motivation for physical fitness or the use of exercise to mitigate stress, and that the moderate gain is due to an increase in muscle mass. It is also possible that the moderate weight gain and improved physical fitness is due to the combination of an increase in exercise and alcohol consumption. The exact etiology is impossible to determine using this data, but our incongruent results suggest additional study is needed.

We investigated the hypothesis that a recent divorce may have a negative link with subsequent deployments. However, we found that those who were recently divorced were more likely to deploy within a 3-year period than those who remained married, suggesting that divorce did not adversely impact deployment eligibility. Recently divorced service members may be more likely to volunteer to deploy as a means to escape emotional hardship, although this study did not collect data on reasons for deployment. The study did not find an association between divorce and separation from the military, suggesting that marital status change may not directly affect the military careers of service members in the short term, but further studies are needed.

The time dependent analysis showed that service members who were recently divorced $(<1$ year) were more likely to report new-onset PTSD, alcohol-related problems, and binge drinking, compared to those who were divorced for $>2$ years. These findings suggest that mental and behavioral health outcomes are more likely to be reported within a shorter time frame, which is consistent with a previous study that found that length of time from marital separation was the most powerful 
factor in emotional resolution [61]. Nonetheless, even short-term health and behavioral conditions demonstrated in this study may interfere with both relationships and work performance, and may influence future behaviors. It is also possible that the symptoms detected by screening instruments in this study were a result of traumatic experiences and processes of grief associated with divorce, but do not represent chronic mental or behavioral health conditions.

Our study has notable limitations. Study outcomes were self-reported and were not validated by medical record review; however, the use of validated surveys to detect mental and behavioral health conditions in a military sample has several advantages because these conditions are often underdiagnosed due to stigma [62]. Although previous studies on the Millennium Cohort found it to be representative, responders were proportionally more likely to be women, educated, and white non-Hispanic $[16,63]$. Because our follow-up period was 3 years and certain outcomes may develop later, we may have had inadequate time to detect these outcomes. Conversely, the effects of outcomes may not be longlasting and may have been underestimated. Analyses were only able to assess temporal proximity as opposed to temporal sequence, and thus we were unable to establish causality. PTSD in particular is a complex affliction that is underreported and may also take variable lengths of time to develop, which may cloud possible associations with divorce. Additionally, the number of legally married study participants is also likely to be conservative in relation to real population of military couples, which includes common law and same-sex marriages. Finally, we did not have data on length of marriage or presence of children, which have been shown to affect the marital relationship [64]. A measure of marital satisfaction at baseline would also have been useful to further elucidate the relationship between divorce and health outcomes.

There are also significant strengths. Few data exist regarding the effects of divorce on physical and mental health outcomes directly related to military readiness and capability. The Millennium Cohort Study represents all branches of the US military and unlike many previous studies, our prospective study was able to assess newonset outcomes. The large sample allowed adequate power for adjustment of many covariates, resulting in more robust comparisons and the ability to detect small differences within subgroups of the study population.

\section{Conclusion}

Recent divorce was associated with screening positive for new-onset PTSD, depression, moderate weight gain, tobacco and alcohol use. Despite these findings, recent divorcees were more likely to be physically active and deploy compared to those who remained married. These data suggest that recently divorced service members should be screened for mental health and behavioral risk factors to improve their overall health and readiness. Given the negative health repercussions of divorce, future studies should identify factors associated with divorce in the military setting to reduce the development of these outcomes.

This work represents report 13-40, supported by the Department of Defense, under Work Unit No. 60002. The views expressed in this article are those of the authors and do not reflect the official policy or position of the Department of the Navy, Department of the Army, Department of the Air Force, Department of Defense, Department of Veterans Affairs, or the US Government. Approved for public release; distribution is unlimited. This research was conducted in compliance with all applicable federal regulations governing the protection of human subjects (Protocol NHRC.2000.0007).

\section{Competing interests}

The authors declare that they have no competing interests.

\section{Authors' contributions}

LW performed the statistical analyses and drafted the manuscript. AS ensured accuracy of statistical analyses and guided in drafting and editing the manuscript. SMW and HM both provided subject matter expertise and critically revised the manuscript. JA provided expertise in statistical analysis. NCC was instrumental in the study: conceived of the study, participated in its design and execution, and was integral in the final revisions of the manuscript. All authors read and approved the final manuscript. The authors affirm that they have listed everyone who contributed significantly to the work in the Acknowledgments.

\section{Acknowledgements}

In addition to the authors, the Millennium Cohort Study Team includes CPT Carrie Donoho, Melissa Frasco, Andrea Ippolito, Isabel Jacobson, Lauren Kipp, Cynthia LeardMann, Gordon Lynch, Sheila Medina-Torne, Christopher Phillips, Kari Sausedo, Emma Schaller, Beverly Sheppard, Donald Slymen, Katherine Snell, Steven Speigle, Daniel Trone, Jennifer Walstrom, John Wesner, Martin White, and James Whitmer, from the Deployment Health Research Department, Naval Health Research Center, San Diego, CA.

We appreciate the support from the Management Information Division, US Defense Manpower Data Center (Seaside, CA, USA); Military Operational Medicine Research Program and US Army Medical Research and Materiel Command (Fort Detrick, MD, USA). In addition, we thank Michelle LeWark from the Naval Health Research Center (San Diego, CA, USA).

\section{Author details}

'Deployment Health Research Department, Naval Health Research Center, San Diego, CA, USA. ${ }^{2}$ Purdue University, West Lafayette, IN, USA. ${ }^{3}$ San Diego State University, San Diego, CA, USA.

Received: 6 October 2014 Accepted: 2 June 2015

Published online: 19 June 2015

\section{References}

1. Hewitt B, Turrell G, Giskes K. Marital loss, mental health and the role of perceived social support: findings from six waves of an Australian population based panel study. J Epidemiol Community Health. 2012;66(4):308-14

2. Rhoades GK, Kamp Dush CM, Atkins DC, Stanley SM, Markman HJ. Breaking up is hard to do: the impact of unmarried relationship dissolution on mental health and life satisfaction. J Fam Psychol. 2011;25(3):366-74. 
3. Choi H, Marks NF. Socioeconomic status, marital status continuity and change, marital conflict, and mortality. J Aging Health. 2011;23(4):714-42.

4. Va P, Yang WS, Nechuta S, Chow WH, Cai H, Yang G, et al. Marital status and mortality among middle Age and elderly Men and women in urban shanghai. PLoS One. 2011;6(11), e26600.

5. Sbarra DA, Law RW, Lee LA, Mason AE. Marital dissolution and blood pressure reactivity: evidence for the specificity of emotional intrusion-hyperarousal and task-rated emotional difficulty. Psychosom Med. 2009;71(5):532-40.

6. Kolves K, Ide N, De Leo D. Marital breakdown, shame, and suicidality in men: a direct link? Suicide Life Threat Behav. 2011:41(2):149-59.

7. Gibb SJ, Fergusson DM, Horwood LJ. Relationship separation and menta health problems: findings from a 30-year longitudinal study. Aust N Z J Psychiatry. 2011;45(2):163-9.

8. Ortega FB, Brown WJ, Lee DC, Baruth M, Sui X, Blair SN. In fitness and health? A prospective study of changes in marital status and fitness in men and women. Am J Epidemiol. 2011;173(3):337-44.

9. Breslau J, Miller E, Jin R, Sampson NA, Alonso J, Andrade LH, et al. A multinational study of mental disorders, marriage, and divorce. Acta Psychiatr Scand. 2011;124(6):474-86.

10. Karney BR, Crown JS. Does Deployment Keep Military Marriages Together or Break Them Apart? Evidence from Afghanistan and Iraq. In: Wadsworth SM, Riggs D, Editors. Risk and Resilience in US Military Families. Springer New York; 2011. p. 23-45

11. Gulf War and Health:Volume 7: Long-Term Consequences of Traumatic Brain Injury. Washington DC: The National Academies Press; 2008

12. Chapin M. Family resilience and the fortunes of war. Soc Work Health Care. 2011;50(7):527-42

13. Returning Home from Iraq and Afghanistan: Assessment of Readjustment Needs of Veterans, Service Members, and Their Families. Washington DC: The National Academies Press; 2013.

14. Riviere LA, Merrill JC, Thomas JL, Wilk JE, Bliese PD. 2003-2009 marital functioning trends among U.S. enlisted soldiers following combat deployments. Mil Med. 2012;177(10):1169-77.

15. Harvey SB, Hatch SL, Jones M, Hull L, Jones N, Greenberg N, et al. The long-term consequences of military deployment: a 5-year cohort study of United kingdom reservists deployed to Iraq in 2003. Am J Epidemiol. 2012;176(12):1177-84

16. Smith TC, Zamorski M, Smith B, Riddle JR, Leardmann CA, Wells TS, et al. The physical and mental health of a large military cohort: baseline functional health status of the Millennium Cohort. BMC Public Health. 2007;7, E340.

17. Gray GC, Chesbrough KB, Ryan MA, Amoroso P, Boyko EJ, Gackstetter GD, et al. The Millennium Cohort Study: a 21-year prospective cohort study of 140,000 military personnel. Mil Med. 2002;167(6):483-8.

18. Ryan MA, Smith TC, Smith B, Amoroso P, Boyko EJ, Gray GC, et al. Millennium Cohort: enrollment begins a 21-year contribution to understanding the impact of military service. J Clin Epidemiol. 2007:60(2):181-91.

19. Jacobson IG, Ryan MA, Hooper TI, Smith TC, Amoroso PJ, Boyko EJ, et al. Alcohol use and alcohol-related problems before and after military combat deployment. JAMA. 2008;300(6):663-75.

20. Smith TC. Linking exposures and health outcomes to a large populationbased longitudinal study: the Millennium Cohort Study. Mil Med. 2011;176(7 Suppl):56-63.

21. Goetzel RZ, Hawkins K, Ozminkowski RJ, Wang S. The health and productivity cost burden of the "top 10" physical and mental health conditions affecting six large U.S. employers in 1999. J Occup Environ Med. 2003;45(1):5-14

22. Weathers FW, Litz BT, HD S. The PTSD Checklist (PCL): Reliability, Validity, and Diagnostic Utility. In: 9th Annual Meeting of the International Society for Traumatic Stress Studies: 1993; San Antonio, Texas. 1993.

23. Blanchard EB, Jones-Alexander J, Buckley TC, Forneris CA. Psychometric properties of the PTSD Checklist (PCL). Behav Res Ther. 1996;34(8):669-73.

24. Brewin CR. Systematic review of screening instruments for adults at risk of PTSD. J Trauma Stress. 2005:18(1):53-62.

25. Wright KM, Huffman AH, Adler AB, Castro CA. Psychological screening program overview. Mil Med. 2002;167(10):853-61.

26. Kroenke K, Spitzer RL, Williams JB. The PHQ-9: validity of a brief depression severity measure. J Gen Intern Med. 2001;16(9):606-13.

27. Spitzer RL, Williams JB, Kroenke K, Linzer M, de Gruy 3rd FV, Hahn SR, et al. Utility of a new procedure for diagnosing mental disorders in primary care. The PRIME-MD 1000 study. JAMA. 1994;272(22):1749-56.
28. Spitzer RL, Kroenke K, Williams JB. Validation and utility of a self-report version of PRIME-MD: the PHQ primary care study. Primary Care Evaluation of Mental Disorders. Patient Health Questionnaire. JAMA. 1999;282(18):1737-44.

29. Spitzer RL, Williams JB, Kroenke K, Hornyak R, McMurray J. Validity and utility of the PRIME-MD patient health questionnaire in assessment of 3000 obstetric-gynecologic patients: the PRIME-MD Patient Health Questionnaire Obstetrics-Gynecology Study. Am J Obstet Gynecol. 2000;183(3):759-69.

30. Jacobson IG, Smith TC, Smith B, Keel PK, Amoroso PJ, Wells TS, et al. Disordered eating and weight changes after deployment: longitudinal assessment of a large US military cohort. Am J Epidemiol. 2009;169(4):415-27.

31. Naimi TS, Brewer RD, Mokdad A, Denny C, Serdula MK, Marks JS. Binge drinking among US adults. JAMA. 2003;289(1):70-5.

32. Stewart AL, Hays RD, Ware Jr JE. The MOS short-form general health survey. Reliability and validity in a patient population. Med Care. 1988;26(7):724-35.

33. Ware Jr JE, Sherbourne CD. The MOS 36-item short-form health survey (SF-36). I. Conceptual framework and item selection. Med Care. 1992;30(6):473-83

34. Saarni SI, Viertio S, Perala J, Koskinen S, Lonnqvist J, Suvisaari J. Quality of life of people with schizophrenia, bipolar disorder and other psychotic disorders. Br J Psychiatry. 2010;197(5):386-94.

35. Wells TS, LeardMann CA, Fortuna SO, Smith B, Smith TC, Ryan MA, et al. A prospective study of depression following combat deployment in support of the wars in Iraq and Afghanistan. Am J Public Health. 2010;100(1):90-9.

36. Forman-Hoffman VL, Peloso PM, Black DW, Woolson RF, Letuchy EM, Doebbeling BN. Chronic widespread pain in veterans of the first Gulf War: impact of deployment status and associated health effects. J Pain. 2007:8(12):954-61.

37. Kline A, Falca-Dodson M, Sussner B, Ciccone DS, Chandler H, Callahan L, et al. Effects of repeated deployment to Iraq and Afghanistan on the health of New Jersey Army National Guard troops: implications for military readiness. Am J Public Health. 2010;100(2):276-83.

38. Lorenz FO, Wickrama KA, Conger RD, Elder Jr GH. The short-term and decade-long effects of divorce on women's midlife health. J Health Soc Behav. 2006;47(2):111-25

39. Sundin J, Jones N, Greenberg N, Rona RJ, Hotopf M, Wessely S, et al. Mental health among commando, airborne and other UK infantry personnel. Occup Med (Lond). 2010;60(7):552-9.

40. Davalos ME, French MT. This recession is wearing me out! Health-related quality of life and economic downturns. J Ment Health Policy Econ. 2011;14(2):61-72.

41. Ross CE, Wu CL. Education, age, and the cumulative advantage in health J Health Soc Behav. 1996:37(1):104-20.

42. Williams DR, Yan Y, Jackson JS, Anderson NB. Racial differences in physical and mental health: socio-economic status. Stress and discrimination. J Health Psychol. 1997;2(3):335-51.

43. Vogt DS, Samper RE, King DW, King LA, Martin JA. Deployment stressors and posttraumatic stress symptomatology: comparing active duty and National Guard/Reserve personnel from Gulf War I. J Trauma Stress. 2008;21(1):66-74

44. Singh JA, Nelson DB, Fink HA, Nichol KL. Health-related quality of life predicts future health care utilization and mortality in veterans with self-reported physician-diagnosed arthritis: the veterans arthritis quality of life study. Semin Arthritis Rheum. 2005:34(5):755-65.

45. Singh JA, Borowsky SJ, Nugent S, Murdoch M, Zhao Y, Nelson DB, et al. Health-related quality of life, functional impairment, and healthcare utilization by veterans: veterans' quality of life study. J Am Geriatr Soc. 2005;53(1):108-13.

46. Butterfield MI, Forneris CA, Feldman ME, Beckham JC. Hostility and functional health status in women veterans with and without posttraumatic stress disorder: a preliminary study. J Trauma Stress. 2000;13(4):735-41.

47. Davila EP, Zhao W, Byrne M, Hooper MW, Messiah A, Caban-Martinez A, et al. Health-related quality of life and nicotine dependence, Florida 2007. Am J Health Behav. 2011;35(3):280-9.

48. Hasin DS, Goodwin RD, Stinson FS, Grant BF. Epidemiology of major depressive disorder: results from the National Epidemiologic Survey on Alcoholism and Related Conditions. Arch Gen Psychiatry. 2005;62(10):1097-106.

49. Kelly SJ, Daniel M, Dal Grande E, Taylor A. Mental ill-health across the continuum of body mass index. BMC Public Health. 2011;11:765.

50. Ewing JA. Detecting alcoholism. The CAGE questionnaire. JAMA 1984:252(14):1905-7.

51. Dhalla S, Kopec JA. The CAGE questionnaire for alcohol misuse: a review of reliability and validity studies. Clin Invest Med. 2007;30(1):33-41. 
52. Amato PR. Research on divorce: continuing trends and New developments J Marriage Fam. 2010;72(3):650-66.

53. Hetherington EM. Intimate pathways: changing patterns in close personal relationships across time. Fam Relat. 2003;52(4):318-31.

54. Karney BR, Crown JS. Families Under Stress: An Assessment of Data, Theory, and Research on Marriage and Divorce in the Military. Santa Monica: RAND Corporation; 2007.

55. Lambert JE, Engh R, Hasbun A, Holzer J. Impact of posttraumatic stress disorder on the relationship quality and psychological distress of intimate partners: a meta-analytic review. J Fam Psychol. 2012;26(5):729-37.

56. Riggs DS, Byrne CA, Weathers FW, Litz BT. The quality of the intimate relationships of male Vietnam veterans: problems associated with posttraumatic stress disorder. J Trauma Stress. 1998;11(1):87-101.

57. Mol SS, Arntz A, Metsemakers JF, Dinant GJ, Vilters-van Montfort PA, Knottnerus JA. Symptoms of post-traumatic stress disorder after non-traumatic events: evidence from an open population study. $\mathrm{Br} J$ Psychiatry. 2005;186:494-9.

58. Menaghan EG, Lieberman MA. Changes in depression following divorce: a panel study. J Marriage Fam. 1986;48(2):319-28.

59. Magura M, Shapiro E. Alcohol consumption and divorce: Which causes which? J Divorce. 1988;12(1):127-36.

60. Lee MR, Chassin L, Mackinnon D. The effect of marriage on young adult heavy drinking and its mediators: results from two methods of adjusting for selection into marriage. Psychol Addict Behav. 2010;24(4):712-8.

61. Gray C, Koopman E, Hunt J. The emotional phases of marital separation: an empirical investigation. Am J Orthopsychiatry. 1991;61(1):138-43.

62. Hoge CW, Auchterlonie $J$, Milliken CS. Mental health problems, use of mental health services, and attrition from military service after returning from deployment to Iraq or Afghanistan. JAMA. 2006;295(9):1023-32.

63. Smith TC, Smith B, Jacobson IG, Corbeil TE, Ryan MA. Reliability of standard health assessment instruments in a large, population-based cohort study. Ann Epidemiol. 2007;17(7):525-32.

64. Grill E, Weitkunat R, Crispin A. Separation from children as a specific risk factor to fathers' health and lifestyles. Soz Praventivmed. 2001;46(4):272-8.

\section{Submit your next manuscript to BioMed Central and take full advantage of:}

- Convenient online submission

- Thorough peer review

- No space constraints or color figure charges

- Immediate publication on acceptance

- Inclusion in PubMed, CAS, Scopus and Google Scholar

- Research which is freely available for redistribution 